\title{
Public Health Measures and the Control of COVID-19 in China
}

\author{
Zhangkai Jason Cheng ${ }^{1} \cdot$ Zhiqing Zhan $^{1,2} \cdot$ Mingshan Xue $^{1} \cdot$ Peiyan Zheng ${ }^{1} \cdot$ Jiali Lyu ${ }^{1} \cdot$ Jing Ma $^{1}$. \\ Xiaohua Douglas Zhang ${ }^{3}$. Wenting Luo ${ }^{1} \cdot$ Huimin Huang ${ }^{1} \cdot$ Yong Zhang ${ }^{1} \cdot$ Hongman Wang ${ }^{4} \cdot$ Nanshan Zhong $^{1}$. \\ Baoqing Sun ${ }^{1}$ (1)
}

Accepted: 30 August 2021 / Published online: 18 September 2021

(c) The Author(s), under exclusive licence to Springer Science+Business Media, LLC, part of Springer Nature 2021

\begin{abstract}
In December 2019, the COVID-19 pandemic quickly spread throughout China and beyond, posing enormous global challenges. With prompt, vigorous, and coordinated control measures, mainland China contained the spread of the epidemic within two months and halted the epidemic in three months. Aggressive containment strategy, hierarchical management, rational reallocation of resources, efficient contact tracing, and voluntary cooperation of Chinese citizens contributed to the rapid and efficient control of the epidemic, thus promoting the rapid recovery of the Chinese economy. This review summarizes China's prevention and control strategies and other public health measures, which may provide a reference for the epidemic control in other countries.
\end{abstract}

Keywords China $\cdot$ COVID-19 pandemic $\cdot$ Infection control and management $\cdot$ Public health

\section{COVID-19 Pandemic}

At the beginning of 2020, the coronavirus disease 2019 (COVID-19) spread rapidly around the world, resulting in a pandemic, thus posing an urgent and severe threat to human health and global peace and development [1]. The outbreak was initially noticed in the largest city in central ChinaWuhan, Hubei Province, which has a population of 11 million,

Zhangkai Jason Cheng, Zhiqing Zhan and Mingshan Xue These authors contributed equally.

Hongman Wang

2496453591@qq.com

$\triangle$ Nanshan Zhong

nanshan@vip.163.com

$\triangle$ Baoqing Sun

sunbaoqing@vip.163.com

1 Department of Allergy and Clinical Immunology, State Key Laboratory of Respiratory Disease, National Clinical Research Center of Respiratory Disease, Guangzhou Institute of Respiratory Health, First Affiliated Hospital of Guangzhou Medical University, Guangzhou, China

2 Guangzhou Medical University, Guangzhou, China

3 Faculty of Health Sciences, University of Macau, Taipa, Macau, China

4 The Fifth Affiliated Hospital of Zunyi Medical University, Zhuhai, China and which serves as a regional economic and transportation hub. As the number of cases rapidly increased, medical resources quickly started to run out, and the initial situation was dire. The discovery of this novel disease and the subsequent epidemic provoked widespread fear in China.

Due to the continuous efforts of Chinese medical workers and authorities, it took only slightly longer than one month after the initial outbreak to contain the spread of the disease and approximately two months to reduce the daily number of new cases in China to fewer than 10 [2]. Since then, China's pandemic prevention and control strategy has shifted to preventing cases from being imported that may lead to a rebound of the local epidemic [3]. The goal of this study is to summarize the prevention, control strategies, and measures adopted by the People's Republic of China during different stages of the COVID-19 pandemic. The Chinese programs and their experience regarding local epidemic prevention and control may provide a reference for other countries and regions during the current global pandemic.

\section{Central Government}

\section{Epidemic Management}

Swift Action Faced with the sudden emergence of the severe acute respiratory syndrome coronavirus (SARS-CoV-2), China quickly implemented appropriate strategies and 
measures in an effort to identify the pathogen and control its spread. The list of actions taken included conducting field epidemiological investigations, identifying and publishing the complete genetic sequence of the virus, designing polymerase chain reaction (PCR) test kits, releasing and constantly updating the COVID-19 clinical guidelines, closely monitoring patients, tracing contacts, and reporting and sharing information with the World Health Organization (WHO). Following the discovery and report of the initial cases on December 29, 2019, the Wuhan Center for Disease Control and Prevention (CDC) conducted a field epidemiological investigation, traced the confirmed cases and their close contacts, ordered the closure of the Huanan Seafood Wholesale Market, and carried out large-scale disinfection campaigns. Hubei Province declared a public health emergency on January 7, 2020, and other provinces quickly followed suit. On January 8, the Wuhan Institute of Virology of the Chinese Academy of Sciences confirmed that the outbreak was caused by a new type of coronavirus, which was later named SARS-CoV-2 [4-6]. On January 10, the Chinese CDC developed the first PCR-based diagnostic kit for COVID-19. On January 15, the National Health Commission (NHC) released the first edition of the COVID-19 clinical guidelines. On January 20, the person-to-person transmission was officially confirmed, and COVID-19 was added to the national class B infectious disease list. Class A infectious disease prevention and control measures were adopted, and direct online reporting was mandated. Due to the top-level emergency response being launched in time, the epidemic growth slowed down and the scale of the epidemic spread was limited.

Centralized Mobilization of National Resources All sectors of China established cooperative relationships with immediate effect. The People's Liberation Army (PLA) and the regional medical institutions organized large-scale medical forces to support Hubei in rapidly improving the healthcare capacity in regions most severely affected by the epidemic. By March 9, 2020, 346 medical teams from across the country, totaling 42,600 people, had arrived in Hubei [7]. In addition, a "province-for-city" pairing assistance strategy, where provinces each pair-matches with one affected city to provide counterpart assistance, was implemented. The medical teams in Wuhan formed a joint expert group to improve overall treatment capacity by combining medical resources [8]. Despite a lack of medical resources in the early stages of the pandemic, China made every effort to save lives. It was the first country to perform lung transplantation in patients with irreversible pulmonary fibrosis caused by COVID-19 [9]. On July 21, 2020, the first COVID-19 lung transplant patient, out of a total of 10 worldwide, was discharged from Renmin Hospital of Wuhan University [10].

\section{Border Control}

To prevent the import of foreign cases, the Chinese government implemented strict immigration control measures such as restricting non-urgent and non-essential cross-border movements of people by not issuing entry documents such as visas. China adopted a 28 -day quarantine and health monitoring program for people entering high-risk areas. After 14 days of centralized quarantine in designated hotels, inbound travelers continued to self-quarantine at home for seven days (for those who did not meet the conditions of home quarantine, hotel quarantine was implemented instead). Health monitoring continued for seven more days after reaching the end of the period.

China implemented a flight circuit breaker mechanism, requiring airlines to suspend flights for two weeks if the number of passengers testing positive after entering China reached five, and four weeks if it reached 10 [11]. Circuit breaker orders were issued to 302 international passenger flights, which was expected to reduce 2,300 imported cases before August 2, 2021 [12]. In addition, various airports in China adopted different measures to restrict passenger flow according to passenger traffic. When the population density reached $0.5-1.0$ per $\mathrm{m}^{2}$, the entry would be controlled; when it reached $1.0-1.5$ per $\mathrm{m}^{2}$, an announcement would be made to remind of the upper limit of indoor people; when it was over 1.5 per $\mathrm{m}^{2}$, no more people would be allowed to enter [13]. In the case of any outbreak in clusters and form chains of transmission, the corresponding airport would close. For example, a small superspreader event occurred at Lukou International Airport in Nanjing, China, on July 21, 2021. Among the regular PCR test samples of airport staff, nine were positive. As of July 25, 101 confirmed cases had been reported in the airport and its associated cases, linking nine cities in five provinces in China. On July 26, 2021, all inbound and outbound flights were successively canceled, and the airport was closed. Furthermore, PCR testing was conducted on all associated airport personnel, followed by at-home quarantine. Coach stations and passenger depots around the airport were temporarily closed [14].

\section{Technological Research}

To combat the pandemic, China increased scientific and technological research in the field of healthcare and launched the COVID-19 pandemic prevention and control emergency project. It mobilized the entire nation to conduct relevant research on clinical treatments, drugs, and vaccines. The Chinese team was the first to share the full genetic sequence of the virus with the WHO, and the first SARS-CoV-2 PCR test kit was commercially released 20 days later, on January 26. The National Center for Biological Information and the 
National Data Center for Microbial Sciences soon released viral genetic sequences and analyses of viral genetic variations [15]. Pathologists in China published the results of the largest series of COVID-19 patient autopsies worldwide, which revealed the presence of SARS-CoV-2 virions in multiple organs in a number of individuals, indicating that there were several routes of viral transmission. The findings of studies on the digestive tract, skin, and various body fluids, for example, facilitated a deeper comprehension of the progression of COVID-19 and the development of more effective interventions [16].

Furthermore, China continuously updated its treatment plan, developed eight versions of the COVID-19 clinical guidelines [17], made the COVID-19 drug screening results publicly available, and provided key treatments, such as favipiravir and convalescent plasma, to countries affected by the pandemic. Chinese scientists immediately launched emergency research projects to investigate technical means of treating severely ill patients, such as artificial liver and blood purification technologies, to improve the treatment success rate in more severe cases [18]. In addition, China used a variety of sophisticated medical equipment to combat the pandemic, such as ventilators, extracorporeal membrane oxygenation (ECMO) machines, $\mathrm{P} 3$ mobile laboratories, and mobile CT, enabling COVID-19 lung transplant patients who had been on ECMO for the longest duration worldwide, to regain spontaneous breathing $[19,20]$.

China established five avenues of vaccine research in the early stages of the pandemic to maximize the success rate: inactivated vaccines, recombinant protein vaccines, adenovirus vector vaccines, attenuated influenza virus vector vaccines, and nucleic acid vaccines. Among them, China developed the world's first inactivated vaccine within 98 days from the start of the research and development to the approval of a clinical trial. The National Drug Administration of China has licensed six SARS-CoV-2 vaccines so far, including four inactivated vaccinations, one adenovirus vector vaccine, and one recombinant protein vaccine [21]. China has licensed 22 SARS-CoV-2 vaccines for clinical studies [22], one of which is an mRNA vaccine now in phase III trials, showing very promising results [23]. In terms of vaccination, China's vaccination program rapidly accelerated after a relatively slow start, and more than 1.9 billion doses of COVID-19 vaccines have been administered to date [24].

\section{Establish Mutual Trust with the Public}

The Chinese government interacted with citizens, established mutual trust, and reached a consensus on the expected actions through the following strategies.

Open and Transparent Information The Chinese government disclosed the epidemic information, providing real-time and dynamic release of data on confirmed, suspected, and cured cases, and deaths across the country. It issued national action plans and employed experts to provide authoritative interpretations, to reduce people's panic and doubts. For example, the Chinese government broadcast the construction of Huoshenshan and Leishenshan hospitals during the outbreak of the pandemic, which attracted 60 million views, reflecting the efficient execution ability and enhancing the credibility of the government.

A Series of Livelihood Protection Measures The Chinese government took a series of measures to protect people's livelihood and ensure the availability of basic public services while implementing the aggressive containment strategy. For example, community workers were arranged point-to-point to supply essential commodities to those quarantined at home. Multi-pronged measures were taken, including reducing corporate burdens, keeping payrolls stable, and creating more jobs. The government provided subsidies for industries and enterprises significantly affected by the epidemic (such as accommodation and catering, sports and recreation, transportation, and tourism industries). When the pandemic was brought under control, dynamic containment strategies were implemented and regions resume work and normal life.

\section{Contact Tracing}

Contact tracing was one of the key tools used for containing viral transmission and identifying close contacts in a timely manner. It was utilized to break the chain of transmission, thus effectively preventing outbreaks in clusters due to individual cases. Different countries utilize different contact tracing technologies, which can be split into three categories: contact tracing based on GPS or location data, Bluetooth technology, and base station data. The adoption of contact tracing technology is compared by countries in Table 1.

Application of Health QR Code The color-based health QR code system was a major Chinese innovation in the field of contact tracing [25]. The health QR code system tracked the contact based on GPS positioning technology, and was mainly used to classify people at different levels of transmission risk through the collection of basic information of residents (such as ID card, and residential address), epidemic prevention information (such as symptoms, vaccination information, and PCR test results), and travel information (such as destination, mode of travel, residence, and travel history). Figure 1 shows the information collected by health QR codes. Authorities issued guidelines mandating the use of health QR codes when entering prominent places such as hospitals, supermarkets, and subways. The health QR code system enabled authorities to quickly locate potentially 


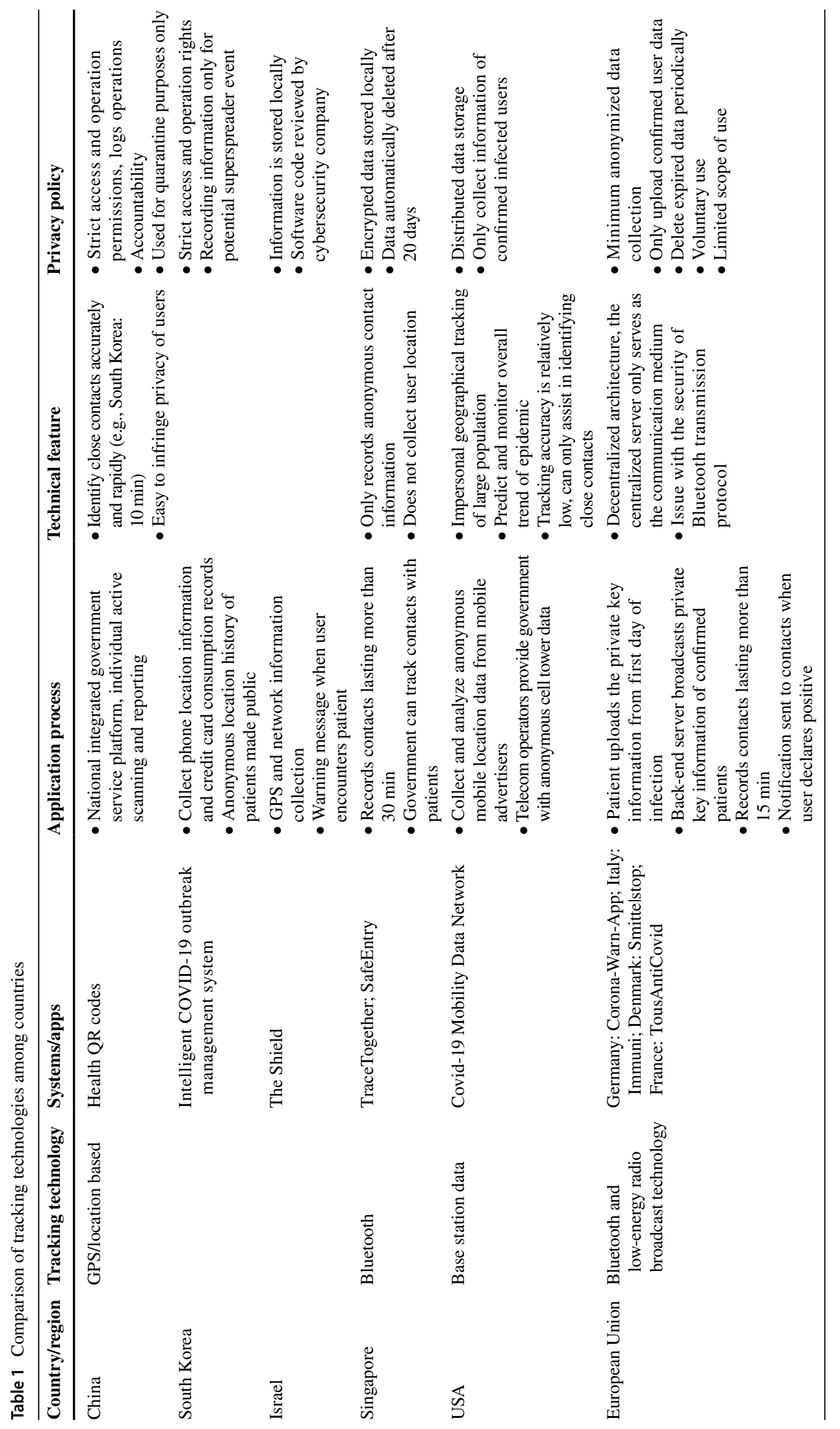


Fig. 1 The information collected by health QR codes

\author{
$<$ Report your health informati... \\ *Health condition \\ Normal \\ fever above $37.3^{\circ} \mathrm{C}$ (including) \\ Dry cough \\ Fatigue \\ Congested or runny nose

\section{*Types of visitors}

Stay in Guangzhou for over 3 months.

Arrived in/returned to Guangzhou $>14 \mathrm{da}$ ys but $<3$ months

Came or returned to Guangzhou within 1 4 days

Currently not in Guangzhou but planning to visit/return to Guangzhou
$<$ Report your health informati... $\quad \cdots \quad \odot$ *Current address

If you do not live in Guangzhou, please enter the address of your work, school, hospital, or other destinations.

\section{Recent contact history (optio nal)}

Feel that you have contacted someone who is infected with COVID-19 within 14 days.

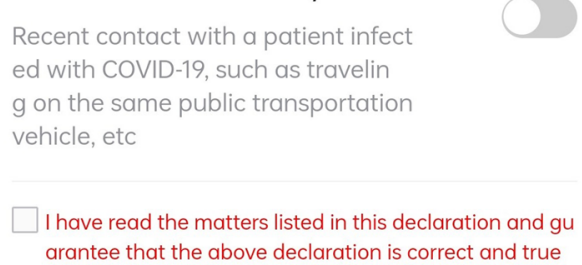

infected people and implement control measures in a timely manner [26]. Figure 2 shows the generation process of a health QR code.

Although health QR codes were not identical in different provinces, three colors-green, yellow, and red-were used to represent different groups of people. The green codes were used for indicating people who were not exposed to the environment exposed to SARS-CoV-2 and could move freely. The yellow codes were used for indicating those who were exposed to the environment exposed to SARS-CoV-2 within the past 14 days. Users were quarantined for seven days, and the codes of the healthy ones were changed to green. The red codes were used for indicating those individuals in close contact with the confirmed cases. Users were quarantined for 14 days, and the codes of the healthy ones would change to green. In order to prevent the use of screenshots for evading testing, the current location and time were added to the interface, to ensure the presentation of realtime and dynamic information. In addition, the application interface also provided a vaccination appointment service. Figure 3 shows the screenshots of the health QR code and its application interface.
However, this technology can easily violate users' privacy. To this end, China has implemented a number of privacy protections, including the data minimization principle, promising that every effort will be made to improve the security of data acquired, establishing an accountability protection mechanism, and enacting necessary legislation to prevent data misuse. The public reacted well to the health code application, and it was soon marketed [27]. At present, almost one billion netizens in China have their own health codes. Within one year of health QR codes coming into service, the cumulative number of users exceeded one billion, and the cumulative number of codes displayed exceeded 24 billion. The health QR code system was successfully launched in more than 20 provincial administrative districts, covering over 400 cities and counties [28]. The application of health QR codes facilitated accurate and effective contact tracing, which allowed people to return to work, thus easing the strain imposed by pandemic control measures on society [29]. According to an online survey, the Chinese public recognizes the health code with an approval rating of 8.49 out of 10 , and more than $90 \%$ of respondents believe that the health code will play a larger role in health, transportation, and other fields in the future [27]. 
Fig. 2 The generation process of a health QR code
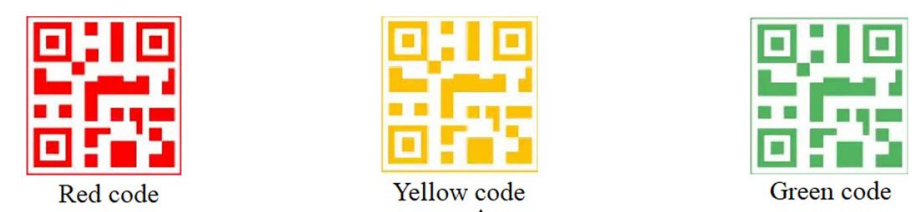

Yellow code

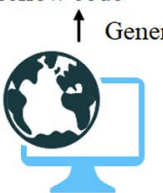

Data is centralized stored and analyzed

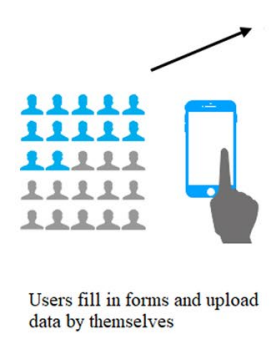

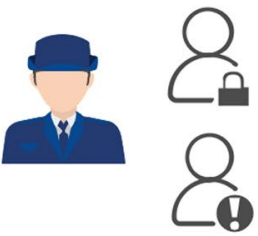

Government sector
Investment in the Resources in Contact Tracing In addition to the health QR code system, China launched services for providing nationwide data about pandemic prevention and control risks. These were based on the relevant departments in all provinces which could inquire from the unified database about confirmed and suspected cases, close contacts,
Fig. 3 The screenshots of the health QR code and its application interface
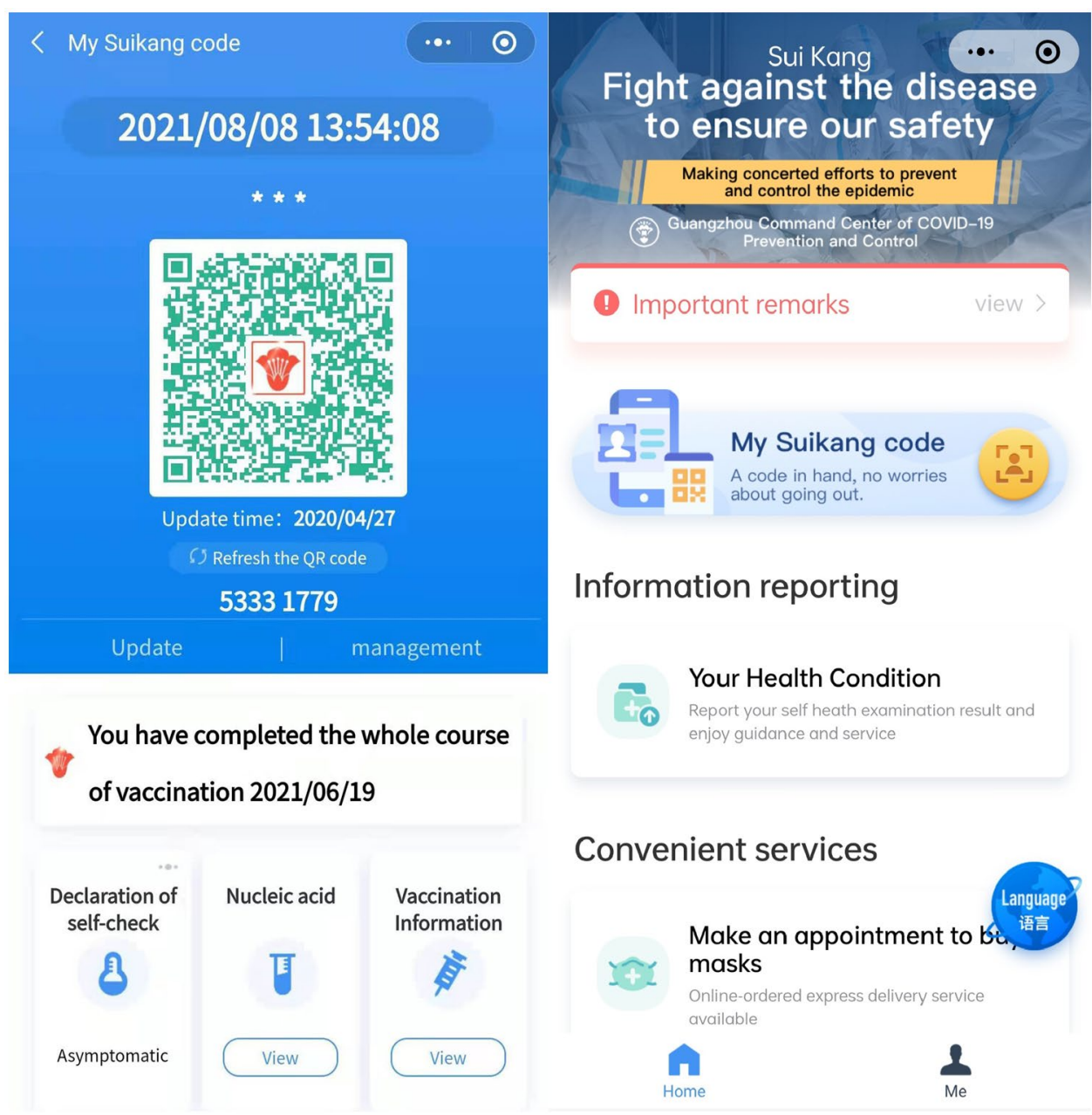

(a)

\section{Convenient services}

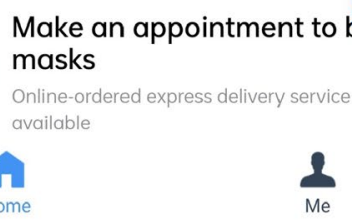

(b) 
and the country-risk level database, realizing sharing and mutual recognition of data. Local governments have also invested a lot of resources in contact tracing. Take Guangzhou as an example, since the first local case in Guangzhou on May 21, 2021, the government conducted inspection and testing, epidemiological investigations, and epidemic prevention and control. Guangzhou allocated more than 60,000 police and mobilized 39 teams (three persons in each team) of epidemiological investigation personnel in Guangzhou and from surrounding cities. These teams determined the trajectory of transmission and conducted on-site surveys and inquiries using information technology. They identified the possible sources of infection and the affected population and more than 1.3 million residents were independently investigated [30].

\section{Municipal Levels}

\section{Outbreak Stage}

Strict Closed-Off Management With interregional population movements, the likelihood of bidirectional transmission and the spread of COVID-19 [31] increased. A closedoff management strategy to limit population flow was a critical early prevention and control strategy adopted. On January 14, 2020, the Wuhan municipal government implemented a policy regarding the departure of personnel and established temperature detection and investigation points at the airport. Wuhan was officially placed under lockdown on January 23 [32, 33], and the closure of national roads, waterways, and passenger flights into and out of Wuhan was announced. Except for essential personnel, all residents were urged to remain at home while dedicated community workers attended to the needs of each household. According to a study, the Wuhan travel ban was associated with a delayed arrival time of COVID-19 in other cities by an estimated 2.9 days, providing extra time to prepare for the arrival of COVID-19 in more than 130 cities across China [34].

Municipal governments in unaffected cities also implemented strict control measures to minimize mass gatherings and stockpiled pandemic prevention materials. Non-essential venues that were sites of public gatherings, such as bars, theaters, and sports stadiums, were ordered to close temporarily. Temperature checkpoints were established in most public areas, including shopping malls and train stations. When someone was suspected to be infected, they and their close contacts would be immediately placed in temporary isolation and detained for testing. They would then be transferred to the appropriate institution designated by the local health administration department.
Designated Treatment by Category It has been reported that most COVID-19 patients with mild symptoms recovered with proper medical care [34]. Therefore, Wuhan constructed a three-tiered management system: designated hospitals were selected to treat severely ill patients. Makeshift hospitals were built to treat and isolate patients with mild and suspected cases. The construction of the makeshift hospitals continued around the clock, and it took just 10 days to build 12 makeshift hospitals, providing more than 20,000 beds [35]. COVID-19 screening was performed among close contacts and febrile patients in communities [36]. In only four days, PCR testing of all clinically confirmed cases, suspected cases, close contacts, and febrile patients in Wuhan had been performed [37]. A total of 9,899,828 people were tested in Wuhan between May 14 and June 1, of whom none had confirmed symptomatic cases of COVID-19, and 300 $(0.303 / 10,000$ population) had asymptomatic infections [38].

\section{Prevention and Control During Periods of Stability}

The number of cases in China was essentially brought under control by April 29, 2020, due to the government's strong prevention and control measures and the coordinated efforts across the country. Although sporadic flare-ups occurred, they were quickly and effectively contained. At this point, the prevention and control strategies employed by provinces and cities shifted to preventing COVID-19 from re-entering the country to cause a new outbreak [39].

\section{In the Absence of Confirmed Cases}

Outbreak Prevention Municipal governments implemented all-around outbreak prevention management, which includes the establishment of quarantine stations at ports of entry, centralized isolation facilities, and other community preventive and control measures. They convened expert committees to issue technical guidelines and work plans in a timely manner, such as for cold chain food production and management during the epidemic, as well as for preventive overall disinfection. They also monitored and improved the efficiency of medical teams. The public was instructed to keep their hands clean, wear masks, reduce the number and size of congregations, and maintain social distancing.

Graded Vaccination The population was divided into three categories according to the risk of exposure and the risk of infection: high-risk population (medical and epidemic prevention personnel, border and port workers, and those in high-risk areas), susceptible population (the elderly, children, pregnant, and those with underlying diseases), and the general population. Sequential vaccination is a necessary measure for overall management and control to relieve the pressure of vaccine production in a short period of time. 
High-risk employees were prioritized to receive vaccination [40].

\section{In the Case of Small-scale Outbreaks}

Control the Source of Infection and Trace the Transmission Chain The source of infection was located through highquality epidemiological investigation, to identify and cut the chain of transmission. Cases were tracked and managed using big data technology, and pandemic-related information was released in a timely manner [39]. A "pandemic map" was also created showing the exact locations, distances, and numbers of infected persons in each community, thus assisting the public in avoiding regions of high risk of infection [41]. Animal monitoring in the closed and controlled areas was actively carried out, to identify other possible sources of infection.

Targeting Risk Areas and Possibly Infected Populations Due to the low viral load, if the sample analyzed is in the early stages of SARS-CoV-2 infection, it may go undetected. As a result, large-scale and successive rounds of PCR testing are favorable to rapid screening of at-risk areas and prompt the adoption of targeted control measures to halt the spread of the epidemic.

Region-Specific, Multi-level Targeted Approach The prevention and control units were demarcated and the risk level was determined according to the changes in the epidemic pattern. Closed-off management areas and control areas were identified according to the risk level, so as to implement different management measures.

Guangzhou, for example, completely contained the epidemic of the Delta variant within 29 days without a lockdown, and the number of the infected were controlled under 153 days [42]. After the outbreak, Guangzhou immediately conducted high-quality epidemiological investigations and performed viral genetic testing of all patients with COVID19 , followed by complete genetic sequencing. Based on risk level, Guangzhou strategically implemented several rounds of PCR tests for nearly 19 million permanent residents and completed the largest-scale nucleic acid screening around the world. It implemented a region-specific, multi-level targeted approach to epidemic prevention and control, based on which, two high-risk areas and 16 medium-risk areas were identified. Guangzhou revised the definition of "close contacts" to "the people who came in contact with a patient four days before the onset in the same space, the same unit, the same architecture, and the same building," based on which, Guangzhou adopted different prevention and control management models for key intensive risk groups. People at different levels of risk of spreading the disease were accurately classified with health $\mathrm{QR}$ codes, and the color markings facilitated management and precise monitoring of at-risk populations. To reduce the risk of spread by the movement of people leaving the city, the green $\mathrm{QR}$ code and a negative PCR test report within $72 \mathrm{~h}$ must be provided prior to departure. Rat cages were set in high-risk areas and 44 valid throat swab specimens were collected from rats captured. Negative PCR test results ruled out the possibility of spread in closed and controlled areas through animal excrement.

\section{Dynamic Hierarchical Control Measures}

Dynamic prevention measures and control at different tier levels are undertaken as a major strategy during the stable period of the epidemic [43]. According to the epidemic situation and population flow, each province is divided into low-, medium-, and high-risk regions. A low-risk region refers to a region without confirmed cases for 14 consecutive days. These regions are fully opened, with health codes used for dynamic monitoring and preparations made for emergencies. A medium-risk region referred to a region with new confirmed cases in 14 consecutive days and if the number of cumulative confirmed cases is below 50. All such areas with positive cases would be locked down locally, and epidemiological investigations and tracking of close contacts would be performed. In addition, there would be restrictions imposed against large gatherings. A high-risk region refers to a region with over 50 cumulative confirmed cases and outbreaks in clusters within the past 14 days. Full lockdown management would be implemented, such as production shutdown and school suspension, and low- and medium-risk regions would mobilize to provide assistance. This strategy allows communities to gradually recover normal life and promoted economic and social development while preventing and controlling the epidemic. The prevention and control measures for each risk level region are shown in Table 2.

\section{Grid Management for a Matrix of Urban Communities}

Communities served as the foundation for efforts to control the pandemic and the first line of defense for vulnerable populations. They adopted grid management-in each grid, working staff were arranged, including administrative staff, staff of the residents' committees, police, and volunteers. Community workers registered entry and exit, investigated households, disinfected venues, and monitored body temperature $[41,44]$. For those returning from affected regions, the community workers registered them and conducted a medical examination, after which they were quarantined at home for 14 days [45]. During city closures 
Table 2 Summary of China's anti-epidemic strategies and measures

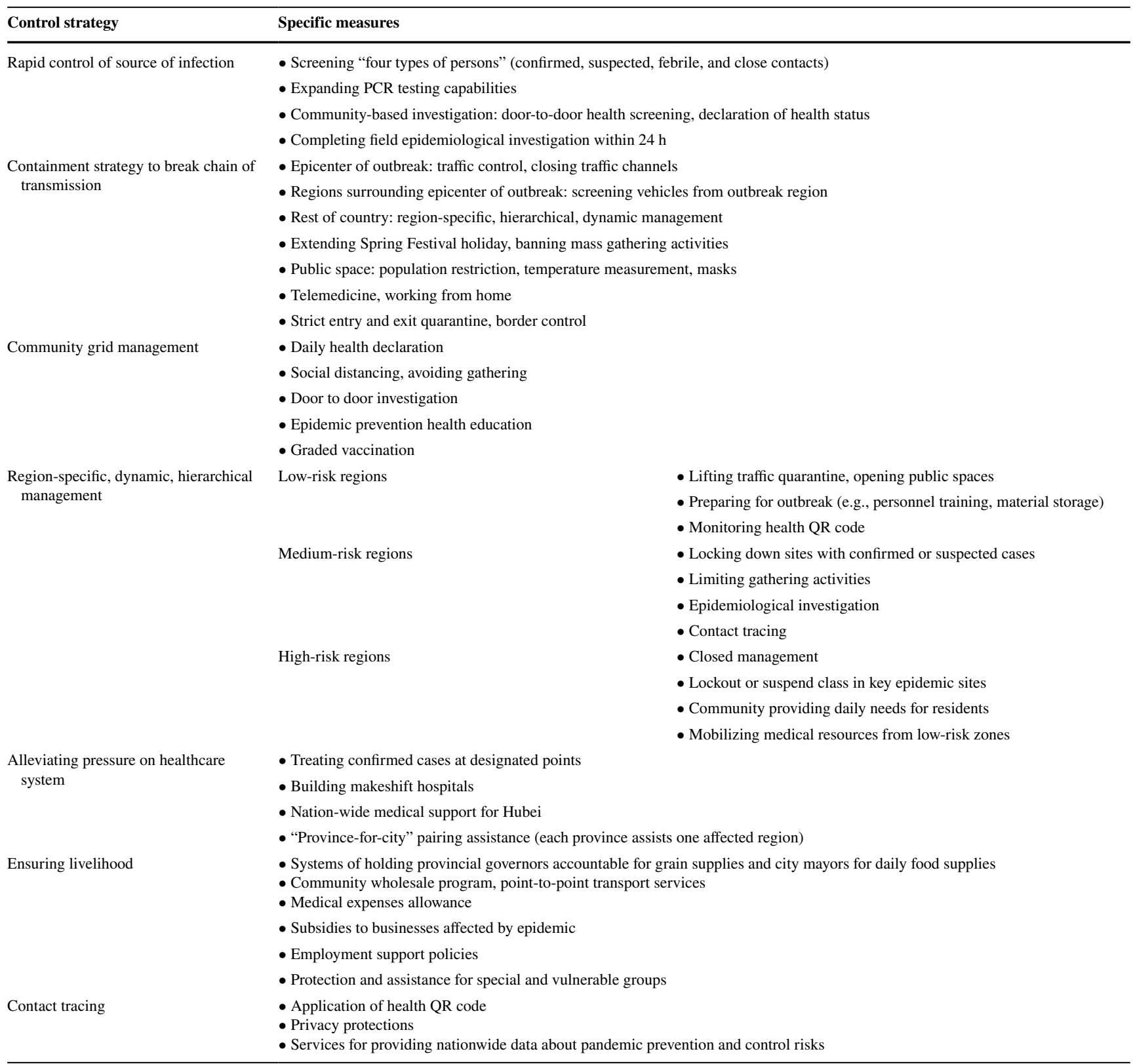

and community lockdowns, systems of holding provincial governors accountable for grain supplies and city mayors for daily food supplies were implemented to maintain an overall balance in the market for daily necessities. In addition, communities also adopted wholesale programs and provided point-to-point transport services for their residents by delivering daily necessities and garbage disposal. Community healthcare centers provided health checks with diagnoses and treatment services. The healthcare center staff members performed door-to-door health screening. Following the preliminary diagnosis and screening of individuals with symptoms, patients who met the criteria for treatment were transferred to the designated hospitals, while the rest were isolated and monitored at home, thus alleviating the strain on hospital resources to a large extent [46].

\section{Private Sector and Individuals}

\section{Support from Private Sectors}

During the COVID-19 pandemic, companies and individuals were valuable resources who contributed greatly to the fight against the pandemic using their strengths and expertise. The number of patients continued to increase in the early days of the outbreak, as did the demand for medical supplies. To 
minimize contact among individuals and reduce the possibility of cross-infection, technological companies developed robots to operate in hospital isolation areas, remote consultation platforms, and remote visiting systems for COVID-19 patients [47-51]. The remote consultation platforms enabled expert panels to remotely evaluate each patient's treatment plan based on their condition, answer questions asked by the designated hospital, and provide professional guidance [52]. Oil companies adjusted their production plans to produce raw materials for medical supplies, such as polyolefin for medical masks and sodium hypochlorite for medical disinfectants. Textile mills worked around the clock to produce medical protective equipment. A navigation company launched the COVID-19 Map, which displayed pandemicrelated information, including travel restrictions, pandemic trends, and the location of vaccination sites.

\section{Cooperation from Citizens}

Although the outbreak of COVID-19 began during the Chinese Spring Festival, the public voluntarily canceled their visits to relatives and friends to reduce interpersonal contact [53]. Chinese people actively responded to the recommendations made by government authorities, including wearing masks and maintaining social distancing, demonstrating a strong sense of responsibility for their own and the health of others $[54,55]$. Although most people supported and cooperated with prevention and control measures, there were still individuals who refused to accept the PCR testing, which could have harmed the prevention and control management. Therefore, it is worth noting that most of the prevention and control measures were mandatory and the citizens who did not cooperate with health reporting and anti-pandemic management faced legal liabilities. For example, people who refused nucleic acid testing were fined, their health codes were turned from green to yellow, and their access to public places was restricted. Those who concealed, lied, and forged personal health information, or organized large gatherings, were held accountable by law.

\section{Discussion}

After the outbreak of COVID-19, China adopted an "aggressive containment" strategy, which helped the government to effectively control the pandemic in a short period of time. The strategy specifically involved: (I) Rapid response for controlling the sources of infection. At an early stage of the pandemic, China quickly identified and controlled the sources of infection through high-quality epidemiological investigations, large-scale PCR testing, and home investigations. (II) Strict closed-off management for cutting off the chain of transmission. Through lockdown of the severely affected area-Wuhan - a region-specific, and region-specific, multi-level targeted approach to epidemic prevention and control was implemented around China. In addition, strict immigration control measures were also implemented (such as the closure of airports and circuit breaker mechanism for airlines). (III) Grid management for a matrix of urban communities. The streets and communities in each city were divided into "grids" according to certain standards to achieve segmented management, which made community-based pandemic prevention management more refined and comprehensive. (IV) Dynamic, precise, and hierarchical prevention and control. After the effective control of the pandemic, the hierarchical management strategies improved the accuracy and efficacy of pandemic prevention and control and minimized the impact on economic and social development and people's livelihood. (V) Centralized mobilization of national resources. The patients with COVID-19 were classified and admitted to designated hospitals based on the severity of infections. Strategies such as "province-for-city" pairing assistance effectively improved the efficiency of treatment and optimized the distribution and supply of medical resources. (VI) Efficient contact tracing. The health QR code system, which served as an efficient contact tracing tool, enabled authorities to group cases, suspected outbreaks, and chains of transmission and helped the authorities make decisions regarding alert levels and other actions. Table 2 lists anti-pandemic strategies and measures taken in China.

\section{Aggressive Containment}

Since the outbreak of COVID-19, countries around the world have responded to the COVID-19 crisis in widely disparate ways. The WHO Independent Panel for Pandemic Preparedness and Response (IPPPR) categorized the pandemic response strategies of different countries as aggressive containment, suppression, or mitigation. China primarily used an active containment strategy against COVID-19 [56].

Despite having more than 80,000 confirmed cases nationwide, China effectively controlled the pandemic within two months (from January 20, 2020, to March 10, 2020), allowing residents to quickly resume work and school [57] with the implementation of a series of aggressive containment strategies. The Chinese government learned from past major disease outbreaks and respected the scientific guidance. Using advanced medical and information technologies, suspected cases were instantly identified [58-61]. Designated isolation facilities were provided for those unable to isolate themselves [62], and community grid management strategies 
were implemented. Information about the pandemic was released in a timely manner, and cooperation with the international community in an open, transparent, and responsible manner. According to a study, without the Wuhan travel ban or the national emergency response, there would have been 744,000 confirmed COVID-19 cases outside Wuhan by day 50 of the epidemic. But together and interactively, these control measures limit the number of confirmed cases reported to $29,839,96 \%$ fewer than expected in the absence of interventions [34]. In the short term, aggressive containment strategies impose burdens on residents, but rapidly achieved control over the pandemic. This was conducive to the quick recovery of society and the economy, prioritizing people's safety and health to the greatest extent possible. Public health and social measures policies on COVID-19 recommended by the WHO and issued by 10 countries during the early stages of the pandemic (January to May 2020) are listed in Table 3.

\section{Active Response of the Public}

As shown in Table 3, during the fight against the pandemic, China established stricter prevention and control policies and measures than most other countries. Those policies were supported and accepted by the public. A detailed survey conducted in 19 countries showed that the Chinese people were most satisfied with the measures to deal with the pandemic taken by the government [63]. According to another survey, China got top scores among approximately 12,500 respondents from 23 economies who ranked their governments' coronavirus responses across four key indicators: national political leadership, corporate leadership, community, and media [64]. Eighty-five percent of Chinese citizens believe the nation will emerge stronger from the crisis. The Chinese government's success in garnering popular trust and confidence can be credited to a strong social consensus developed by the government and the general public in the fight against the pandemic. Since the outbreak, the Chinese government has issued timely, open, and transparent information about the outbreak, as well as fully informed the public about the epidemic's latest development and the fight against it through live broadcast press conferences. To improve the public's scientific awareness of the epidemic, scientists are responsible for delivering interviews to the media and releasing authoritative views on scientific epidemic prevention to the public. Authoritative scientific research institutions are in charge of interpreting epidemic data and anti-epidemic policies, with full public knowledge as a precondition for the establishment of effective communication and interaction between the government and the public, so that the public can understand the government's epidemic policies in a timely and comprehensive manner. On the other hand, the government has taken measures to ensure people's livelihoods. Systems of holding provincial governors accountable for grain supplies and city mayors for daily food supplies have been implemented to maintain an overall balance in the market for daily necessities. Community wholesale programs, point-to-point transport services, and free psychological intervention services have been provided during the closed-off management. Policies have also been promulgated to assist businesses, stabilize employment, and create more jobs.

In addition, the high degree of public cooperation is related to the Chinese culture and national experience. Chinese believe that personal interests are based on collective interests. When the government announced that $83 \%$ of clusters were family-based, local people meeting the conditions for admission voluntarily admitted themselves in the makeshift hospitals for centralized isolation. Although they were separated from their families and friends, they understood that isolation protected their families and friends from infection. Earlier in 2003, when China had experienced SARS, a series of anti-epidemic laws and policies were formulated. In the early days of the COVID-19 outbreak, the immediate response from the Chinese government strengthened people's trust in the government.

\section{International Collaboration}

COVID-19 is not restricted by national borders and no country is immune to the pandemic. Since the outbreak of the pandemic, China has actively increased its international technical cooperation and engagement in exchanges regarding pandemic prevention and control. During the early stages of the pandemic, many foreign governments, international organizations, and civil societies donated medical supplies and necessary materials to China. A total of 79 nations and 10 international organizations donated masks, protective clothing, and other urgently needed pandemic prevention and control materials to China [65]. Improvements in domestic pandemic prevention and control capacity enabled China to in turn provide materials, technologies, and experience-based assistance to other countries affected by the pandemic. Medical teams, for example, were dispatched to Pakistan, Italy, Iran, and other countries [66], and relevant materials were distributed to 89 countries and four international organizations. Professional and technical training, information, and epidemiological and clinical data were shared with foreign public health and medical institutions. China collaborated with the international community in the search for the origins of COVID-19, effective drugs and vaccines, and useful methods of detection [67], and shared the complete genetic sequence of the virus with the WHO [68]. 


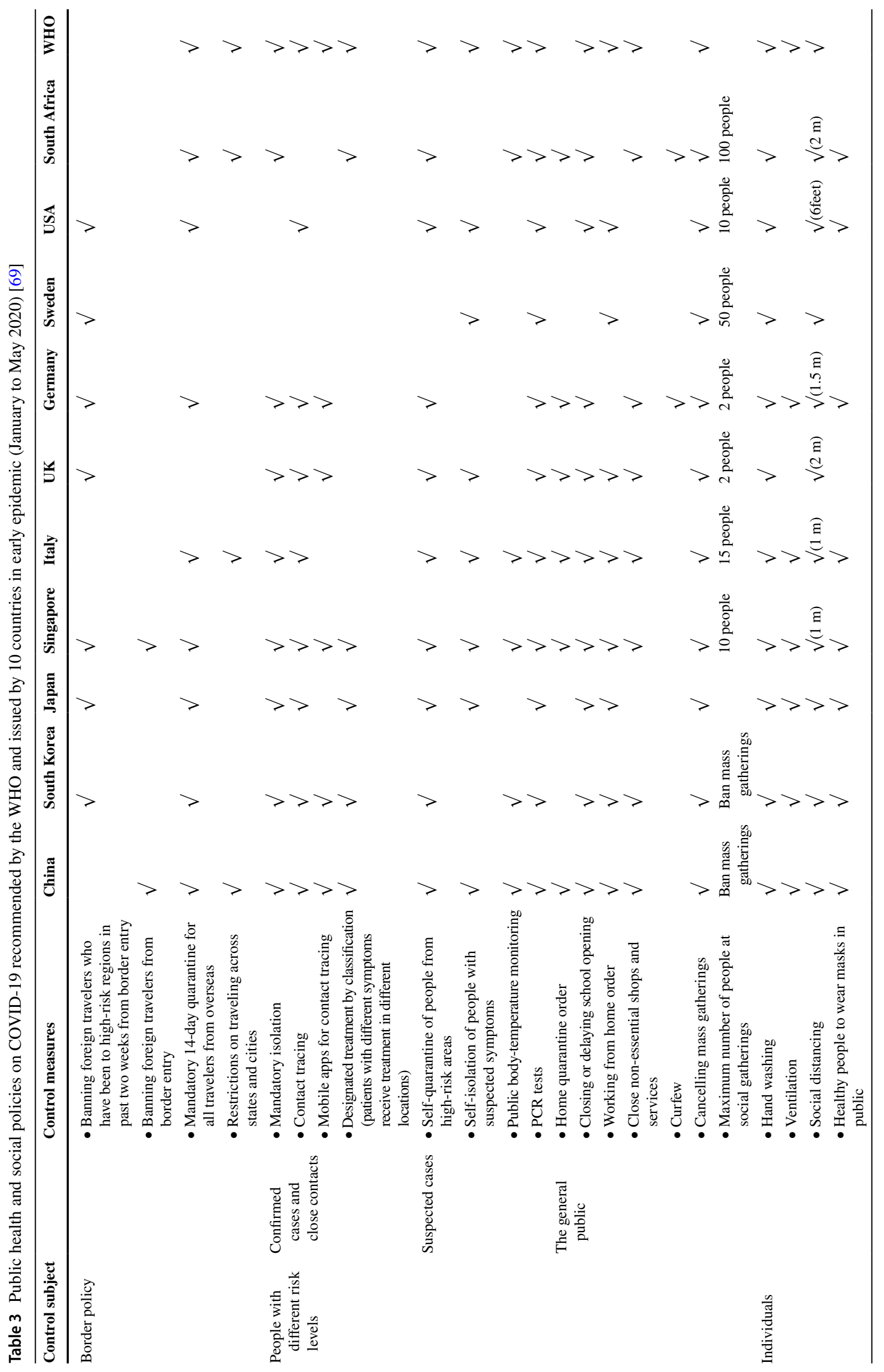




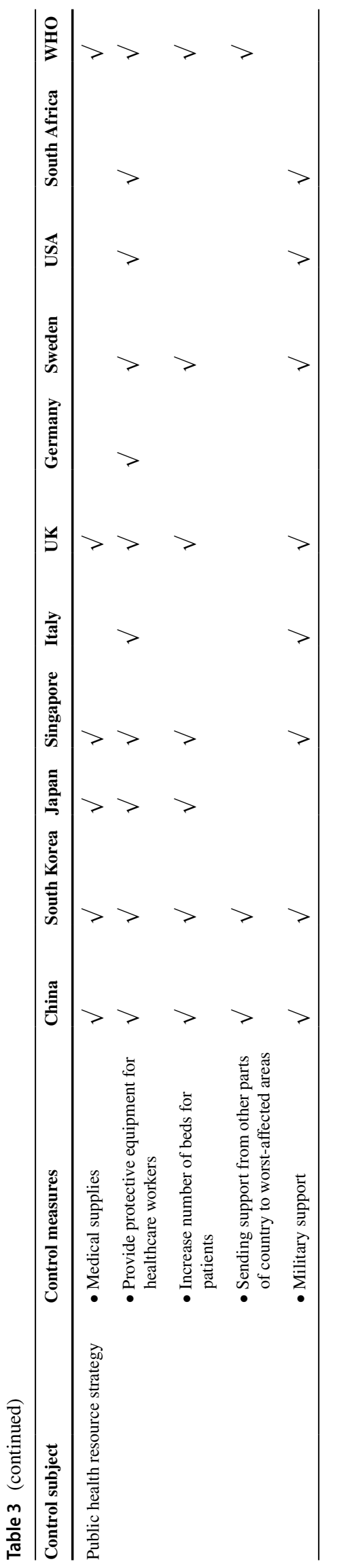

\section{Concluding Remarks}

China's pandemic response has demonstrated the nation's ability to unite and coordinate, from the level of individuals to communities, local governments, and the central government. This coordination and cooperation have played a decisive role in protecting the entire nation from the pandemic and in quickly restoring industry. During the COVID-19 outbreak, the Chinese government quickly implemented measures that had a significant impact on the control and prevention of the disease. There has not been a single protest in China against COVID-19 control measures. During the worst phase of the pandemic, the government imposed a citywide lockdown, requiring all residents to stay at home to prevent the spread of COVID-19, while other parts of the country strictly restricted travel. The general public actively supported and strictly adhered to these measures.

As the pandemic became more serious in some countries, those governments adopted some anti-pandemic measures, such as those pertaining to restricting mass gatherings, maintaining social distancing, and staying at home, but widespread support and strict compliance on the part of the citizens were lacking. Large protests against government restrictions took place in the UK, Italy, Australia, and other countries. In addition, in some countries, the implementation of quarantine and tracking technology sparked considerable debate. It was feared that these measures would result in the restriction of personal freedom and invasion of personal privacy.

It is important to note that the policies implemented in one country may be difficult to replicate in another. Different countries have different cultures and experiences. China has experienced the epidemic outbreak of SARS and has already implemented epidemic preparedness plans and legislations. Thus, China could react quickly to the COVID-19 pandemic. The level of coordination between the government and the citizens may also impact the effectiveness of pandemic preparedness measures. During an outbreak, the Chinese government establishes mutual trust with the public through a series of measures (such as the timely release of fully open and transparent information, use of network media to defuse public anxiety and fear, and safeguard measures to protect people's livelihood), resulting in society-wide consensus on outbreak response measures, promoting civic consciousness. As a result, the Chinese populace is highly supportive of the government's tough restrictions. In addition, people in China prioritize collective interests over personal interests, and believe that individual freedom is only possible through the realization of the good of the group. When a conflict arises between public health and personal privacy, Chinese citizens are more likely to prioritize public health and trust the government to protect their privacy to 
the greatest extent possible. As a result, when drawing on China's experience with combatting the pandemic, it may be necessary to consider each country's political, sociocultural, and environmental factors to evaluate the effectiveness of these measures.

Funding This study was supported by the Guangzhou Institute of Respiratory Health Open Project (Funds provided by China Evergrande Group) (Project No.: 2020GIRHHMS04), and the Zhongnanshan Medical Foundation of Guangdong Province (Project No.: ZNSA-2021005 and Project No.: ZNSA-2020001), and the State Key Laboratory of Respiratory Disease, Guangdong-Hong Kong-Macao Joint Laboratory of Respiratory Infectious Disease.

\section{Declarations}

Conflict of Interest The authors declare no competing interests.

\section{References}

1. Cheng ZJ, Shan J (2020) 2019 Novel coronavirus: where we are and what we know. Infection 48(2):155-163. https://doi.org/10. 1007/s15010-020-01401-y

2. China's action against COVID-19 (2020) The state council information office of the people's republic of china. http://www.gov.cn/zhengce/ 2020-06/07/content_5517737.htm. Accessed 20 August 2021

3. Zhao J, Yuan Q, Wang H et al (2020) Antibody responses to SARSCoV-2 in patients with novel coronavirus disease 2019. Clin Infect Dis 71(16):2027-2034. https://doi.org/10.1093/cid/ciaa344

4. Ren LL, Wang YM, Wu ZQ et al (2020) Identification of a novel coronavirus causing severe pneumonia in human: a descriptive study. Chin Med J (Engl) 133(9):1015-1024. https://doi.org/10. 1097/CM9.0000000000000722

5. Epidemiology Working Group for NCIP Epidemic Response, Chinese Center for Disease Control and Prevention (2020) The epidemiological characteristics of an outbreak of 2019 novel coronavirus diseases (COVID-19) in China. Zhonghua Liu Xing Bing Xue Za Zhi 41(2):145-151. https://doi.org/10.3760/cma.j.issn. 0254-6450.2020.02.003

6. Zhou P, Yang XL, Wang XG et al (2020) A pneumonia outbreak associated with a new coronavirus of probable bat origin. Nature 579(7798):270-273. https://doi.org/10.1038/s41586-020-2012-7

7. Mei C, Ding P (2020) Ministry of National Defense of the People's Republic of China. Nearly 3,000 beds have been set up in the army, and more than 10,000 medical staff have been sent to the front line for treatment. http://www.mod.gov.cn/topnews/2020-03/ 03/content_4861388.htm. Accessed 20 August 2021

8. Zhu Y (2020) The Central Committee of the Communist Party of China and the State Council of China. National Health Commission: Coordinated arrangements for 19 provincial counterparts to support 16 cities and counties in Hubei except Wuhan. http:// www.gov.cn/xinwen/2020-02/11/content_5477116.htm. Accessed 20 August 2021

9. Han W, Zhu M, Chen J, Zhang J, Zhu S, Li T, Cai H, Fang Q, Wei G, Liang T (2020) Lung transplantation for elderly patients with end-stage COVID-19 pneumonia. Ann Surg 272(1):e33-e34. https://doi.org/10.1097/SLA.0000000000003955

10. Xinhua Net (2020) First in the world! Coronal lung transplant patient discharged from hospital! https://baijiahao.baidu.com/s? $\mathrm{id}=1672806092125471877 \& \mathrm{wfr}=$ spider $\&$ for $=$ pc. Accessed 20 August 2021

11. Civil Aviation Administration of China (2020) Civil Aviation Administration adjusts international passenger flight circuit breaker measures http://www.caac.gov.cn/XXGK/XXGK/TZTG/ 202012/t20201216_205607.html. Accessed 20 August 2021

12. Beijing News (2020) Over the past year, civil aviation has fused 302 flights, and it is expected to reduce the number of imported cases by more than 2300 https://www.sohu.com/a/481205197_ 114988. Accessed 20 August 2021

13. Civil Aviation Administration of China (2020) Technical guidelines for epidemic prevention and control at transport airports (Seventh Edition) http://www.gov.cn/xinwen/2021-02/09/5586231/files/ 03ea2343f9d444a38a4f1874b37e935d.pdf. Accessed 20 August 2021

14. Beijing News (2020) Nanjing: all-round closure management of Lukou Airport https://baijiahao.baidu.com/s?id=1706364939027534812\& wfr $=$ spider\&for $=$ pc. Accessed 20 August 2021

15. Lu R, Zhao X, Li J et al (2020) Genomic characterisation and epidemiology of 2019 novel coronavirus: implications for virus origins and receptor binding. Lancet 395(10224):565-574. https:// doi.org/10.1016/S0140-6736(20)30251-8

16. Bian XW (2020) COVID-19 Pathology Team. Autopsy of COVID19 patients in China. Natl Sci Rev 7(9):1414-1418. https://doi. org/10.1093/nsr/nwaa123

17. General Office of the National Health Commission (2021) Notice on the issuance of the COVID-19 protocol (trial eighth revision). http://www.gov.cn/zhengce/zhengceku/2020-08/19/5535757/files/ da89edf7cc9244fbb34ecf6c61df40bf.pdf. Accessed 20 August 2021

18. China Daily (2020) The Second COVID-19 Multidisciplinary Forum on Global Fight Against Epidemic. https://baijiahao. baidu.com $/ \mathrm{s} ? \mathrm{id}=1662463496587959831 \& \mathrm{wfr}=$ spider $\&$ for $=$ pc. Accessed 20 August 2021

19. Meng L, Qiu H, Wan L, Ai Y, Xue Z, Guo Q, Deshpande R, Zhang L, Meng J, Tong C, Liu H, Xiong L (2020) Intubation and ventilation amid the COVID-19 outbreak: Wuhan's experience. Anesthesiology 132(6):1317-1332. https://doi.org/10.1097/ALN. 0000000000003296

20. Ma X, Liang M, Ding M, Liu W, Ma H, Zhou X, Ren H (2020) Extracorporeal membrane oxygenation (ECMO) in critically ill patients with coronavirus disease 2019 (COVID-19) pneumonia and acute respiratory distress syndrome (ARDS). Med Sci Monit 26:e925364. https://doi.org/10.12659/MSM.925364

21. National Medical Products Administration (2021) https://www. nmpa.gov.cn/. Accessed 20 August 2021

22. Xinhua (2021) 22 COVID-19 vaccines enter clinical trials in China: health official. https://www.chinadaily.com.cn/a/202107/ 08/WS60e6e34da310efa1bd660a6f.html. Accessed 20 August 2021

23. Chinese Clinical Trial Registry (2021) A global, multi-center, randomized, double-blind, placebo-controlled, phase III clinical study to evaluate the protective efficacy, safety and immunogenicity of SARS-CoV-2 mRNA vaccine in population aged 18 years and older. https://www.chictr.org.cn/showproj.aspx?proj=130672. Accessed 20 August 2021

24. National Health Commission of the People's Republic of China (2021) Novel coronavirus vaccine inoculation. http://www.nhc. gov.cn/jkj/s7915/202108/afb009eb54eb4fff86c9d9595ecb74f3. shtml. Accessed 20 August 2021

25. Nakamoto I, Wang S, Guo Y, Zhuang W (2020) A QR code-based contact tracing framework for sustainable containment of COVID19: evaluation of an approach to assist the return to normal activity. JMIR Mhealth Uhealth 8(9):e22321. https://doi.org/10.2196/ 22321 
26. Boulos MNK, Geraghty EM (2020) Geographical tracking and mapping of coronavirus disease COVID-19/severe acute respiratory syndrome coronavirus 2 (SARS-CoV-2) epidemic and associated events around the world: how 21 st century GIS technologies are supporting the global fight against outbreaks and epidemics. Int J Health Geogr 19(1):8. https://doi.org/10.1186/ s12942-020-00202-8

27. People's Daily Online (2020) Investigation report of health code. https://baijiahao.baidu.com/s?id=1677590328357483692\&wfr $=$ spider\&for $=$ pc. Accessed 20 August 2021

28. Sina News (2021) First anniversary report of the health code. http://k.sina.com.cn/article_5787187353_158f17899020017ma9. html. Accessed 20 August 2021

29. Tai L, Wong K, Wang L, Di LJ (2020) From impossible to possible: the lessons from the control of recent COVID-19 outbreaks in China. Int J Biol Sci 17(6):1600-1612. https://doi.org/10.7150/ ijbs.58906

30. CCTV news (2021) Guangzhou has invested 60000 police to stop the spread of the epidemic. https://www.sohu.com/a/474011252_ 162522. Accessed 20 August 2021

31. Xiong Y, Wang Y, Chen F, Zhu M (2020) Spatial statistics and influencing factors of the COVID-19 epidemic at both prefecture and county levels in Hubei Province, China. Int J Environ Res Public Health 17(11):3903. https://doi.org/10.3390/ijerph17113903

32. Xu T, Yang R (2020) COVID-19 Epidemic and public health measures in China. J Epidemiol Glob Health 10(2):118-123. https://doi.org/10.2991/jegh.k.200421.001

33. Kickbusch I, Leung G (2020) Response to the emerging novel coronavirus outbreak. BMJ 368:m406. https://doi.org/10.1136/ bmj.m406

34. Tian H, Liu Y, Li Y et al (2020) An investigation of transmission control measures during the first 50 days of the COVID-19 epidemic in China. Science 368(6491):638-642. https://doi.org/10. 1126/science.abb6105

35. Xinhua Net (2020) Wuhan makeshift hospital expanded to 12 planned to open more than 20,000 beds. http://www.xinhuanet. com/2020-02/19/c_1125596243.htm. Accessed 20 August 2021

36. Liu W, Guan WJ, Zhong NS (2020) Strategies and advances in combating COVID-19 in China. Engineering (Beijing) 6(10):1076-1084. https://doi.org/10.1016/j.eng.2020.10.003

37. Haifeng C (2020) Interim measures for prevention and control of pneumonia of new coronavirus infection in Wuhan. http:// www.chinanews.com/gn/2020/01-30/9073042.shtml. Accessed 20 August 2021

38. Health Commission of Hubei Province (2020) The 104th press conference on the prevention and control of novel coronavirus pneumonia epidemic. $2020 \mathrm{http}: / /$ wjw.hubei.gov.cn/bmdt/ ztzl/fkxxgzbdgrfyyq/xxfb/202006/t20200603_2376649.shtml. Accessed 20 August 2021

39. Shen J, Duan H, Zhang B et al (2020) Prevention and control of COVID-19 in public transportation: experience from China. Environ Pollut 266(Pt 2):115291. https://doi.org/10.1016/j.envpol. 2020.115291

40. CCTV New (2020) National Health Commission: high risk groups and high-risk groups will be given priority to vaccinate COVID-19 vaccine. https://baijiahao.baidu.com/s?id=1681057433403193310\& $w f r=$ spider\&for $=$ pc. Accessed 20 August 2021

41. Miao Q, Schwarz S, Schwarz G (2020) Responding to COVID-19: community volunteerism and coproduction in China. World Dev 137:105128. https://doi.org/10.1016/j.worlddev.2020.105128

42. Nanshan Zhong (2021) Guangzhou summed up five experiences in dealing with SARS-CoV-2 variant Delta for the first time. https://m. thepaper.cn/baijiahao_13322445. Accessed 20 August 2021

43. State Council of the PRC (2020) Guiding opinions on COVID-19 prevention and control work in the area of scientific prevention and control of precise policies. http://www.gov.cn/xinwen/202002/18/content_5480514.htm. Accessed 20 August 2021

44. State Council of the PRC (2020) Notice on strengthening community prevention and control of pneumonia epidemic of new coronavirus infection. http://www.gov.cn/xinwen/2020-01/27/ content_5472481.htm. Accessed 20 August 2021

45. The People's Govemment of Guangzhou Municipality (2020) Circular of Guangzhou headquarters for prevention and control of pneumonia epidemic caused by novel coronavirus infections on further strengthening community and rural epidemic prevention and control (no. 3). http://www.gz.gov.cn/xw/tzgg/content/post_ 5655563.html. Accessed 20 August 2021

46. Department of Primary Healthcare of the PRC (2020) Primary healthcare institutions' key role in epidemic control. http://www.nhc.gov. cn/jws/s3578/202002/19c2e477e8c04e20b76730daeb1296f8.shtml. Accessed 20 August 2021

47. Sina News (2020) Guangdong People's Hospital enables 5G infrared temperature measurement with accuracy to \pm 0.2 degrees $\mathrm{Cel}$ sius. http://news.sina.com.cn/s/2020-02-06/doc-iimxxste9254472. shtml. Accessed 20 August 2021

48. Hangzhou Daily (2020) The nation's first 5 G medical temperature patrol robot came to work in Zhejiang Province. http://www.hangzhou. gov.cn/art/2020/2/7/art_812262_41886452.html. Accessed 20 August 2021

49. China Daily (2020) Guangxi's first $5 \mathrm{G}$ thermal imaging temperature measuring device put into use in Qinzhou. http://ex.chinadaily.com. $\mathrm{cn} /$ exchange/partners/82/rss/channel/cn/columns/80x78w/stories/ WS5e3d0e93a3107bb6b579dd2b.html. Accessed 20 August 2021

50. He D, Gu Y, Shi Y, Wang M, Lou Z, Jin C (2020) COVID-19 in China: the role and activities of Internet-based healthcare platforms. Glob Health Med 2(2):89-95. https://doi.org/10.35772/ ghm.2020.01017

51. China National Health Commission (2020) Notice of the General Office of the National Health Commission on internet diagnosis and treatment consulting services in epidemic prevention and control. http://www.nhc.gov.cn/yzygj/s7653p/ 202002/ec5e345814e744398c2adef17b657fb8.shtml . Accessed 20 August 2021

52. Gong K, Xu Z, Cai Z, Chen Y, Wang Z (2020) Internet hospitals help prevent and control the epidemic of COVID-19 in China: multicenter user profiling study. J Med Internet Res 22(4):e18908. https://doi.org/10.2196/18908

53. Zanin M, Xiao C, Liang T, Ling S, Zhao F, Huang Z, Lin F, Lin X, Jiang Z, Wong SS (2020) The public health response to the COVID19 outbreak in mainland China: a narrative review. J Thorac Dis 12(8):4434-4449. https://doi.org/10.21037/jtd-20-2363

54. Fan S, Wu M, Ma S, Zhao S (2020) A preventive and control strategy for COVID-19 infection: an experience from a third-tier Chinese city. Front Public Health 8:562024. https://doi.org/10. 3389/fpubh.2020.562024

55. Yang X, Chen X, Ding C, Bai Z, Zhu J, Sun G, Yu G (2020) Epidemiological investigation and prevention control analysis of the longitudinal distribution of COVID-19 in Henan Province. China mSphere 5(5):e00867-e920. https://doi.org/10.1128/mSphere.00867-20

56. Lancet $\mathrm{T}$ (2020) Sustaining containment of COVID-19 in China. Lancet 395(10232):1230. https://doi.org/10.1016/S0140-6736(20) 30864-3

57. Pan A, Liu L, Wang C, Guo H, Hao X, Wang Q, Huang J, He N, Yu H, Lin X, Wei S, Wu T (2020) Association of public health interventions with the epidemiology of the COVID-19 outbreak in Wuhan. China JAMA 323(19):1915-1923. https://doi.org/10. 1001/jama.2020.6130

58. Zhong N, Zeng G (2006) What we have learnt from SARS epidemics in China. BMJ 333(7564):389-391. https://doi.org/10. 1136/bmj.333.7564.389 
59. Enserink M (2003) SARS in China. China's missed chance Science 301(5631):294-296. https://doi.org/10.1126/science.301.5631.294

60. Xinhua News Agency (2020) Use big data to strengthen accurate measures for epidemic prevention and control. http://www.gov.cn/ xinwen/2020-01/27/content_5472436.htm. Accessed 20 August 2021

61. State Administration of Science, Technology and Industry for National Defence, PRC (2020) The close contact meter of COVID-19 patients has been launched. http://www.sastind.gov. cn/n112/n117/c6808819/content.html. Accessed 20 August 2021

62. Ministry of Health (2003) Measures for prevention and control of infectious SARS. http://www.gov.cn/banshi/2005-08/01/content_ 19099.htm. Accessed 20 August 2021

63. Lazarus JV, Ratzan S, Palayew A et al (2020) COVID-SCORE: a global survey to assess public perceptions of government responses to COVID-19 (COVID-SCORE-10). PLoS One 15(10):e0240011. https://doi.org/10.1371/journal.pone.0240011

64. Blackbox Corp (2020) World in Crisis Global News Coverage. https://blackbox.com.sg/?news-feature=world-in-crisis-gloabalnews-coverage. Accessed 20 August 2021

65. Song W (2020) China's global engagement to fight the novel coronavirus pandemic. Glob Health Res Policy 5:44. https://doi.org/ 10.1186/s41256-020-00172-1
66. Jin H, Lu L, Liu J, Cui M (2021) COVID-19 emergencies around the globe: China's experience in controlling COVID-19 and lessons learned. Int J Qual Health Care 33(1):mzaa143. https://doi. org/10.1093/intqhe/mzaa143

67. Zhou Q (2020) International collaboration for global accessibility of COVID-19 vaccines. Natl Sci Rev 7(8):1269. https://doi.org/ $10.1093 / \mathrm{nsr} / \mathrm{nwaa} 147$

68. Xinhua Net (2020) China shares genetic sequence of novel coronavirus from Wuhan: WHO. http://www.xinhuanet.com/english/ 2020-01/13/c_138700521.htm. Accessed 20 August 2021

69. Wang D, Mao Z (2021) A comparative study of public health and social measures of COVID-19 advocated in different countries. Health Policy 125(8):957-971. https://doi.org/10.1016/j.healthpol. 2021.05.016

Publisher's Note Springer Nature remains neutral with regard to jurisdictional claims in published maps and institutional affiliations. 